\section{Diode molecules}

from our Molecular Physics Correspondent

Not many years ago a distinguished theoretical chemist deliberately left the field, explaining that an important factor in his decision to work elsewhere was the strong possibility, as he conceived it, that no further, totally new and astonishing phenomenon might ever again be discovered at the purely molecular level. Premature as this may have been at the time, and counterindicated as it may seem by subsequent events-for example in the area of laser photochemistry-it remains broadly true that, in spite of remarkable advances in instrumentation and the mastering of known effects in ingenious ways, the incidence of altogether new ones in the molecular world could be said to have lagged behind the steady stream of named, prizewinning, conference-engendering effects in other subjects. Having said which, one may as reasonably remark that refuge from pessimism can be sought in the illdefined yet compelling suspicion that what does remain to emerge from the fastnesses of undiscovered molecular behaviour may prove to be very spectacular indeed.

A sound conjecture-because in a way a truism-is that radically new effects are likely to involve some form of electromagnetic, probably timedependent phenomenon. Stimulated emission fitted this description; the widely publicised speculations about molecular superconductivity are a long shot in the same direction where the merest vestige of an effect would be altogether sensational.

Closer to Earth, yet of undoubted promise, is the recent speculation by Arieh Aviram and Mark Ratner (Chem. Phys. Lett., 29, 277; 1974) that it should be possible to design molecular rectifiers by careful patching together of suitable donor and acceptor subsystems. The key to this idea is that, by bridging an acceptor unit, perhaps a quinone-like ring system, by a $\sigma$-bonded hydrocarbon bridge to an electrophobic unit, perhaps containing methoxy-groups, the necessary currentvoltage asymmetry could be achieved. Actually more exotic candidates for donor and acceptor are available and might be tailor-made for the task, with the passive bridge playing an essential part both in providing rigidity and keeping donor and acceptor at arm's length -just out of range of direct levelinteractions, though not of tunnelling transfer.

No doubt these bare ideas will need considerable testing and elaboration before it can be claimed that rectifier action is a practical possibility. But, showing admirable willingness to back their level diagrams with some real mathematics, Aviram and Ratner go on to present a careful perturbational calculation of the current-voltage characteristics of a plausible rectifier structure (actually a simple hemiquinone). This is semi-quantitative quantum mechanics at its best, using relatively crude approximations to warrant an idea and point to further refinements. When the necessary matrix-elements are computed, with reasonable assumptions of ionisation energy and electron affinity, the I-V characteristic is indeed of the form predicted, with an appreciable asymmetric barrier to conduction.

Needless to emphasise, far too much is left to good fortune for the results to approach a theoretical 'proof' of viable rectifier action. Direct electrodeelectrode tunnelling could swamp the whole effect; the complex problems of metal-organic junctions and crystal field effects have yet to be built ineach might either enhance or kill off the desired effect. Yet the work described has a convincing air of the premier pas qui coûte and we may well hear a lot more of the molecular rectifier, perhaps the absolute ultimate in electronic miniaturisation.

\section{Volcanoes and ice ages}

by John Gribbin

AcCording to Kennett and Thunell (Science, 187, 497; 1975) "volcanic ash in deep-sea sections indicates very high rates of explosive volcanism during the last two million years". This apparent global increase in Quaternary explosive volcanism flies in the face of some cherished geophysical beliefs-but it offers a new insight into the widespread and frequent glacial activity that has occurred over the same period.

Simple tectonic theory would suggest that volcanic activity is related to local events-collisions between blocks of crust which lead to mountain building. But it now seems from study of 320 deep-sea sections drilled around the world during the Deep Sea Drilling Project that there has been a much higher rate of explosive volcanism from both island arc and 'hot spot' volcanoes during the past 2 million years than the 'normal' level of the past 20 million years. And, as Kennett and Thunell point out, "increased Quaternary volcanism coincides approximately with that episode of the Cenozoic marked by major and rapidly fluctuating climatic change".

Just how these two effects may be related remains to be unravelled, but several interesting possibilities are raised. First, it is well established that under appropriate conditions the spread of volcanic dust in the atmosphere caused by a marked increase in volcanic activity could trigger the onset of an ice age. The dust simply acts as a veil blocking some of the Sun's heat, and if the resulting cooling lasts for long enough for ice cover to become established it may take more than the removal of the dust veil to melt the ice, because the ice's high albedo will continue to ensure that little solar heat is absorbed.

But studies of shorter stretches of the geological record than the 20 million year sample of the DSDP show that although widespread volcanic activity often precedes the spread of the glaciers it is possible either for glaciation to occur without any increase in volcanic activity, or for there to be an increase in volcanism without immediate climatic repercussions. This encourages speculation that there are one or more additional mechanisms of key importance in triggering ice ages. The Milankovitch model, in which phases of cooling are produced by changes in the orientation of the Earth and by 'stretch' of its orbit around the Sun is perhaps the most attractive of these mechanisms, especially in the form in which Kukla has recently presented it (Nature, 253, 600; 1975). To my mind, the most plausible overall model of ice ages is that when the northern hemisphere is at its coolest phase of the astronomical Milankovitch cycle, conditions will be appropriate for ice cover to develop dramatically if some further effectsuch as volcanic activity-provides the final trigger.

But the model can also be turned on its head. The repeated loading and unloading of the Earth's crust caused by the advance and retreat of the glaciers might, in fact, trigger increased volcanic activity. And, if each of these processes occurs there could be a snowball effect by which a small climatic fluctuation becomes a full-scale ice age.

Whatever the truth of the matter, these new discoveries cmphasise the impossibility of trying to deal with problems in the earth sciences within rigid compartments. The development of plate tectonic theory has already contributed greatly to the study of past climates by explaining how the present state of the globe, with a nearly landlocked North Polar Ocean, differs from the situation in earlier times when the continents were distributed differently and ice ages were extremely uncommon (see page 14 of this issue of Nature). And at a more immediately important level, it seems that a better understanding of volcanism, as well as a better understanding of ocean currents and other aspects of earth science, will be an essential prerequisite to a really satisfactory theory of climatic change. 